\title{
Unilateral Hypoglossal Nerve Palsy Following the Use of the Laryngeal Mask Airway
}

\author{
Timothy S. Lo
}

Can. J. Neurol. Sci. 2006; 33:320-321

Hypoglossal nerve injury is a rare complication of anesthetic airway management. We report a case of unilateral hypoglossal nerve injury following the use of the laryngeal mask airway (LMA). A 48-year-old man with no past medical history sustained a proximal humerus fracture. His pre-operative examination was normal except for a humerus fracture. Anesthesia was induced and a size-3 LMA was inserted successfully on first attempt without difficulty. The cuff was inflated with the recommended $20 \mathrm{ml}$ of air until there was no leak and maintained at a pressure of $10-15 \mathrm{~cm} \mathrm{H}_{2} 0$. The surgery was uneventful and the duration of anesthesia was two hours. Peri-operatively the patient was hemodynamically stable without episodes of hypotension. The recovery staff noted approximately three hours after the surgery that his tongue was deviated to the left. The patient complained of difficulty swallowing and slurred speech. He had normal sensation of his tongue, taste was

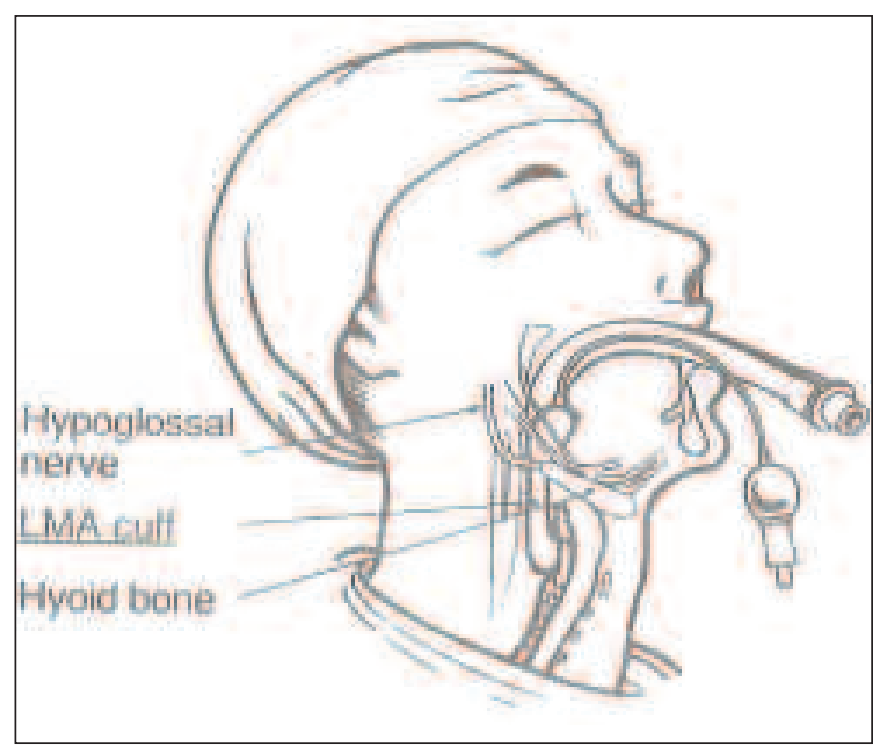

Figure: The hypoglossal nerve and the LMA: the hypoglossal nerve passes just behind the greater cornu of the hyoid bone. A distended LMA cuff may compress the hypoglossal nerve against the hyoid bone. Reprinted with permission from Blackwell Publishing, Oxford, England. ${ }^{2}$ unchanged, and the gag reflex was intact. No other cranial nerves were involved and no other neurological deficiency was found. The following day, there was significantly less dysarthria and slight improvement in tongue movement. Conservative management included speech therapy and regular assessment. He continued to improve and complete recovery took place within two weeks.

In comparison to the endotracheal tube, the LMA has been reported to pose less risk to laryngeal function ${ }^{1}$ and is widely used. To our knowledge, there have only been a few reported cases of unilateral hypoglossal nerve injury ${ }^{2,3}$ after the use of the LMA. These were considered neurapraxic injuries which eventually resolved. Although rare, isolated peripheral nerve injuries following LMA use may cause alteration of taste, swallowing and speech. Local compression of the hypoglossal nerve due to malposition of the mask, excessive cuff pressure, and patient positioning were considered to have been the most likely explanation of these isolated hypoglossal nerve injuries.

At the level of the angle of the mandible the hypoglossal nerve becomes superficial and lies just above the greater horn of the hyoid bone before turning medially toward the tongue. The inflated cuff of the LMA could lie in close proximity to the base of the tongue and pyriform fossae. ${ }^{3}$ The probable site of injury is at the greater horn of the hyoid bone where the inflated cuff of the LMA can compress the nerve against the bone.

Other etiologies of isolated hypoglossal nerve palsy have been described. Compression by a kinked vertebral artery (hypoglossal-vertebral entrapment syndrome) and occipital condylar fracture have been implicated in hypoglossal nerve injuries. The hypoglossal nerve may also be injured in isolation in the neck or in its more distal course near the tongue from carotid aneurysms, aneurysms of a persistent hypoglossal artery, vascular entrapment, spontaneous dissection of the extracranial internal carotid artery, local infections, surgical or accidental

From the Neurological Institute, Columbia-Presbyterian Medical Center, New York, New York, USA.

Received February 8, 2005. ACCEPTED in FinAl fORM MARCH 13, 2006 Reprint requests to: Timothy S. Lo, 1619 Fairview St. \#1, Berkley, California, 94703, USA. 
trauma, neck radiation, and tumors of the retroparotid or retropharyngeal spaces, neck, salivary glands, and base of the tongue. ${ }^{4}$

The overall safety of the LMA is attested to by its widespread use. However, disabilities including dysphagia may occur as a complication of LMA use. Understanding the course of the hypoglossal nerve and awareness of these LMA-associated nerve injuries may aid in the diagnosis.

\section{REFERENCES}

1. Lee SK, Hong KH, Chloe H, Song HS. Comparison of the effect of the laryngeal mask airway and endotracheal intubation on vocal function. B J Anaesth. 1993; 71: 648-50.

2. Nagai K, Sakuramoto C, Goto F. Unilateral hypoglossal nerve paralysis following the use of the laryngeal mask airway. Anaesthesia. 1994; 49: 603-4.

3. King C, Street MK. Twelfth cranial nerve paralysis following the use of a laryngeal mask airway. Anaesthesia. 1994; 49: 786-7.

4. Brazis PW, Masdeu JC, Biller J. Localization in clinical neurology. 4th ed. Philadelphia: Lippincott Williams \& Wilkins; 2001. p. 345 . 